\title{
Review of recent forward physics results from the CMS experiment
}

\author{
Ralf Ulrich ${ }^{1, *}$ for the CMS Collaboration \\ ${ }^{1}$ Institute for Nuclear Physics, Karlsruhe Institute of Technology (KIT), 76131 Karlsruhe
}

\begin{abstract}
There is a rich program of forward physics measurements within the CMS Collaboration covering a wide range of topics. In many cases there is a connection to quantities and effects relevant for very high energy cosmic ray interactions. Some of the recent measurements in the fields of exclusive final states, low- $\mathrm{p}_{\mathrm{T}}$ inclusive and diffractive cross sections, underlying event, multiparton interactions, double parton scattering, final state particle correlations and minimum bias results are briefly summarized here.
\end{abstract}

\section{Introduction}

In this proceedings some of the recent results of the forward physics activity of the CMS Collaboration are summarized. It is interesting that these measurements are not just limited to small (forward) angles, but in particular cover also low- $p_{\mathrm{T}}$, exclusive final states and in particular minimum bias results. The type of measurements discussed here cover up to 14 orders of magnitude in cross section, ranging from very rare exclusive $\mathrm{W}^{+} \mathrm{W}^{-}$pair production in proton-proton collisions at $\sqrt{s}=8 \mathrm{TeV}$ with a cross section on the order of $O(10 \mathrm{fb})$ up to the inclusive cross section in proton-lead collisions at $\sqrt{s_{\mathrm{NN}}}=5.02 \mathrm{TeV}$ on the order of $O$ (barn).

Some of the measurements described have a relation to very high energy cosmic ray interactions and can be of use to further improve the understanding of the modelling of those.

\section{CMS and its forward instrumentation}

The CMS Collaboration benefits from a powerful general purpose particle experiment, with good forward instrumentation using dedicated detectors.

At the heart of the CMS detector is a superconducting solenoid of $6 \mathrm{~m}$ internal diameter, providing a strong magnetic field of 3.8T. The data used for this paper were taken in June 2015 during a period without magnetic field. Within the CMS magnet volume are an inner silicon pixel and strip tracker that measure charged particles in the range $|\eta|<2.5$, a homogeneous lead tungstate crystal electromagnetic calorimeter, and a brass and scintillator hadron calorimeter. The corresponding endcap detectors instrument the pseudorapidity range up to $|\eta| \lesssim 3$ with tracking and calorimetry. Forward Cherenkov calorimeters extend the coverage beyond $|\eta| \gtrsim 3$. Muons are measured in gas-ionization detectors embedded in the steel return yoke.

\footnotetext{
*e-mail: ralf.m.ulrich@kit.edu
}

The hadron forward (HF) calorimeters cover the region $2.9<|\eta|<5.2$ and consist of $2 \times 432$ readout towers, each containing a long and a short quartz fiber embedded within a steel absorber running parallel to the beam. The long fibers run the entire depth of the HF calorimeter $(165 \mathrm{~cm}$, or approximately 10 interaction length), while the short fibers start at a depth of $22 \mathrm{~cm}$ from the front of the detector. The response of each tower is determined from the sum of signal in the corresponding long and short fiber.

The very forward angles on one side of CMS $(-6.6<$ $\eta<-5.2)$ are covered by the CASTOR calorimeter. It has 16 azimuthal towers, each built from 14 longitudinal modules. The 2 front modules form the electromagnetic section, and the 12 rear modules form the hadronic section. The calorimeter is made of stacks of tungsten and quartz plates, read out by PMTs, in two half-cylindrical mechanical structures, and is placed around the beam pipe at a distance of $-14.4 \mathrm{~m}$ away from the nominal interaction point. The overall longitudinal depth of both CASTOR and HF corresponds to 10 hadronic interaction lengths.

A more detailed description of the CMS detector can be found in Ref. [1].

\section{Exclusive final states}

The exclusive production of a particular final state in a hadron-hadron collision is only possible if the exchange particle is colour neutral, thus, either a colour neutral hadronic state (i.e. a pomeron), a photon, or a combination of both.

In particular the collisions where a pomeron is involved offer the exciting opportunity to directly probe the low-x part of the gluon distribution in the hadrons. The most prominent of such measurements are the exclusive production of vector mesons. CMS has measured exclusive $\Upsilon$ production in proton-lead collisions at $\sqrt{s_{\mathrm{NN}}}=5.02 \mathrm{TeV}$. In this system the lead nucleus acts as the source for the photons, while the proton for the pomerons. One result of this measurement is the photon-proton cross 


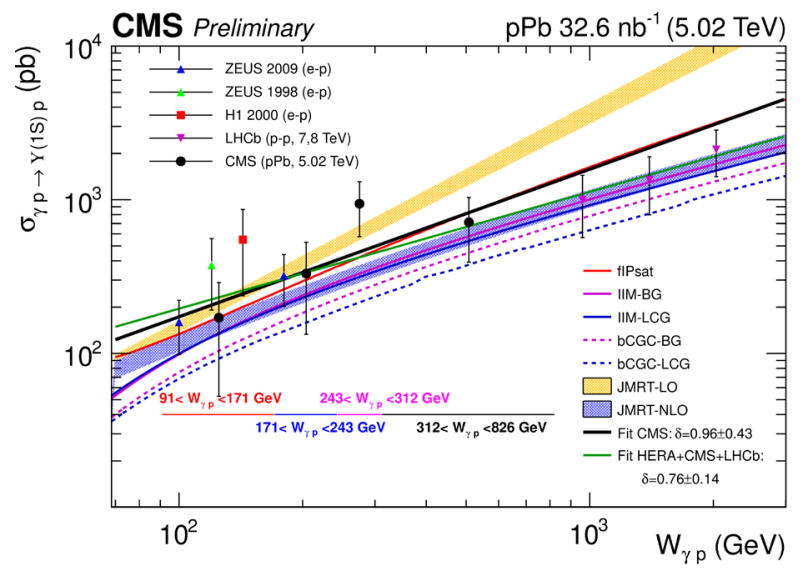

Figure 1. Measurement of the photon-proton cross section as function of photon energy (from Ref. [2]). This result is part of the exclusive $\Upsilon$ measurement by CMS in proton-lead collisions at $\sqrt{s_{\mathrm{NN}}}=5.02 \mathrm{TeV}$.

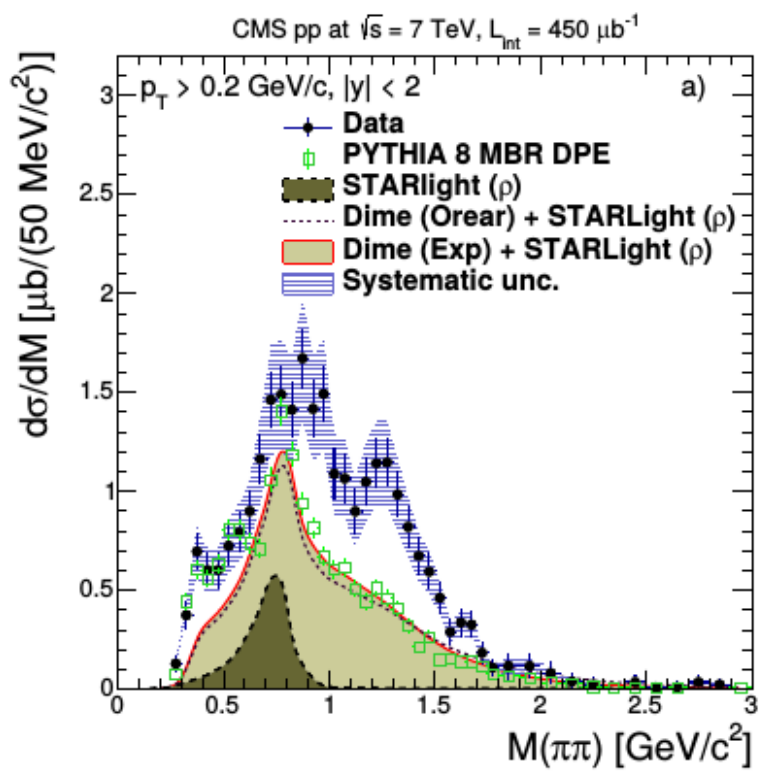

Figure 2. The distribution of the invariant $\pi^{+} \pi^{-}$mass distribution in (semi-) exclusive production, see Ref. [3], measured in protonproton collisions at $7 \mathrm{TeV}$.

section as a function of the photon energy over a much wider range than previously possible, see Fig. 1.

The (semi-)exclusive production of $\pi^{+} \pi^{-}$in protonproton collisions at $7 \mathrm{TeV}$ is a much more complex environment. It contains a continuum distribution, and resonances on top of that (see Fig. 2). The main production mechanism is via diffraction, but with a transition to the regime where one or both of the protons dissociate and produce further particles. These data can reveal information on the probability of proton dissociation in diffractive interactions.

Furthermore, in inelastic collisions where jets are produced it is possible that the two jets are pushed appart from each other by a color singlet exchange (CSE). The

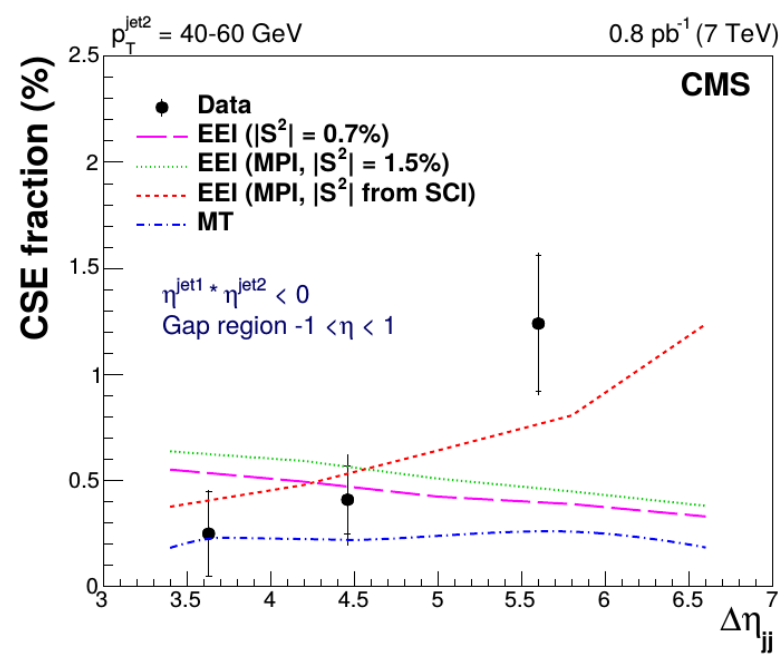

Figure 3. The fraction of colour singlet exchange in events with two jets, see Ref. [4], as function of the jet-separation $\Delta \eta_{j j}$.

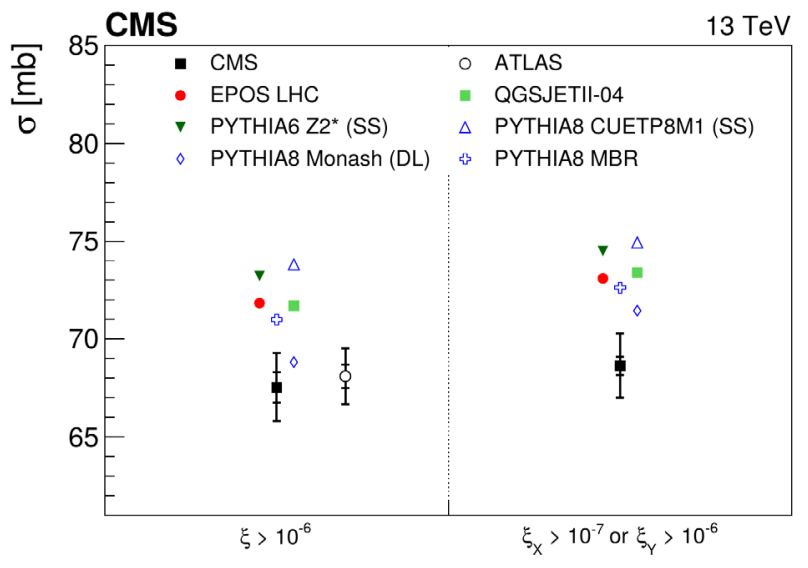

Figure 4. The detector-level inelastic cross section in protonproton collisions measured for $\xi>10^{-6}$ and for $\xi_{x}>10^{-7}$ or $\xi_{y}>10^{-6}$, see Ref. [5]

signature of this is studied in jet-gap-jet measurements in proton-proton collisions at $7 \mathrm{TeV}$, see Fig. 3. These data are useful to search for the signature of BFKL effects.

Another type of collision is where only photons are exchanged. At a hadron collider this can provide an incredibly clean environment for very rare processes. Namely, these is exclusive pair production where e.g. $\mathrm{W}^{+} \mathrm{W}^{-}$are a powerful probe for anomalous quartic gauge coupling and physics beyond the standard model [7]. If this can be combined with the simultaneous observation of the two outgoing proton projectiles the kinematics of the protons can be correlated with the central exclusive system opening up a window for searches for new physics. The CMS-TOTEM Proton Precision Spectrometer (CT-PPS) is built for this purpose [8]. 

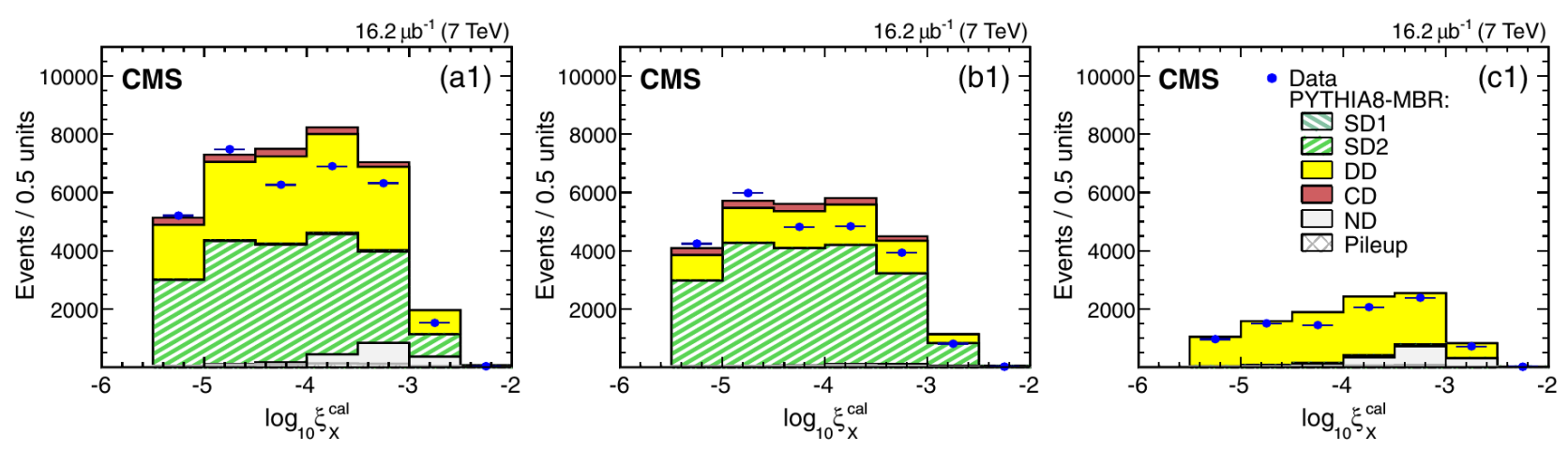

Figure 5. The shapes of the distributions of the number of events as function of the proton rapidity loss as determined by CMS calorimeters $\xi_{x}^{\mathrm{cal}}$. In (a1) all events with forward rapidity gap are shown, in (b1) all of those events with no signal in CASTOR, and in (c1) those events that were flagged by CASTOR. See Ref. [6].

\section{Inclusive cross sections, and diffraction}

One of the most important parameter concerning the development of extensive air shower cascades is the inelastic cross section for particle production. The inelastic cross section in proton-proton collision has been measured at $13 \mathrm{TeV}$ [5]. This analysis is published in two acceptance regions, where the difference is in including the CASTOR very forward calorimeter, see Fig. 4. It is very interesting to see that both measured values are considerably smaller than all model predictions. The CMS measurement at $\xi>10^{-6}$ is consistent with the corresponding measurement by ATLAS. A smaller inelastic cross section will lead to a deeper penetration of air shower cascades.

In the determination of the inelastic cross section, the most difficult problem is to understand the impact of lowmass diffraction. The amount of cross section lost due to detector acceptance is a relatively poorly constrained quantity. In Fig. 5 the measured proton momentum loss, $\xi$, distributions in proton-proton collisions at $7 \mathrm{TeV}$ are shown with different event selections. It can be seen that the separation of single- and double-diffraction is extremely difficult with typical LHC detectors. In CMS, only the inclusion of the CASTOR calorimeter helps to get a better handle on it.

\section{Measurements of inclusive distributions}

The measurement of differential inclusive jet $p_{\mathrm{T}}$ spectra over very wide $p_{\mathrm{T}}$ ranges, shown in Fig. 6, is a very strong test of model predictions. It has been shown that the shape of the parton distribution functions is sensitive to such distributions. In particular the gluon distribution could be improved considering high-precision inclusive jet spectra. The very low-x gluon parton distribution becomes moreand-more important when the centre-of-mass energy increases, thus, it is relevant in very high energy cosmic ray interactions.

In cosmic ray air showers nuclear effects are another aspect that requires precise modelling. The parton distribution functions in nuclear interactions are subject to important further corrections, like shadowing or saturation.

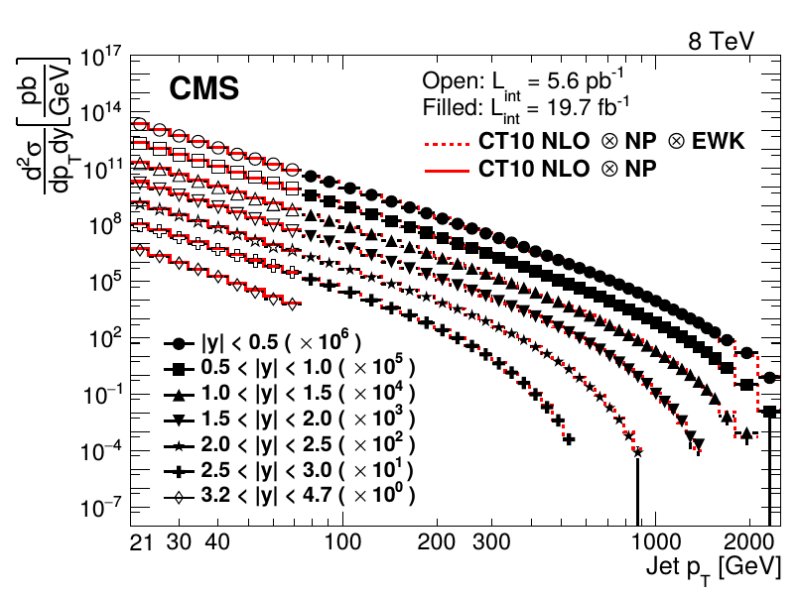

Figure 6. Differential cross sections of inclusive jet production as function of jet $p_{\mathrm{T}}$, see Ref. [9]. The filled markers are measured by all CMS Calorimeters with $\eta<3$, while the open markers by the HF calorimeters.

This will also affect multiparton interactions and the underlying event. For this reason it is very important that CMS measured the very forward inclusive jet cross sections in proton-lead collisions at $\sqrt{s_{\mathrm{NN}}}=5.02 \mathrm{TeV}$ using data of the CASTOR calorimeter, see Fig. 7. These data highlights the large importance of nuclear effects for very high energy cosmic ray interactions.

\section{Underlying Event, multiparton interactions, double parton scattering}

The measurements of the underlying event is a typical experimental method to assess the accuracy of QCD effects in event generation that are very difficult to be determined from first principles. This includes initial- and final-state radiation, multiparton interactions, and projectile fragmentation. The corresponding measurement in proton-proton collisions at $13 \mathrm{TeV}$ is shown in Fig. 8. The EPOS LHC model has some problems to describe the data in the region around jet- $p_{\mathrm{T}}$ of $20 \mathrm{GeV}$. 

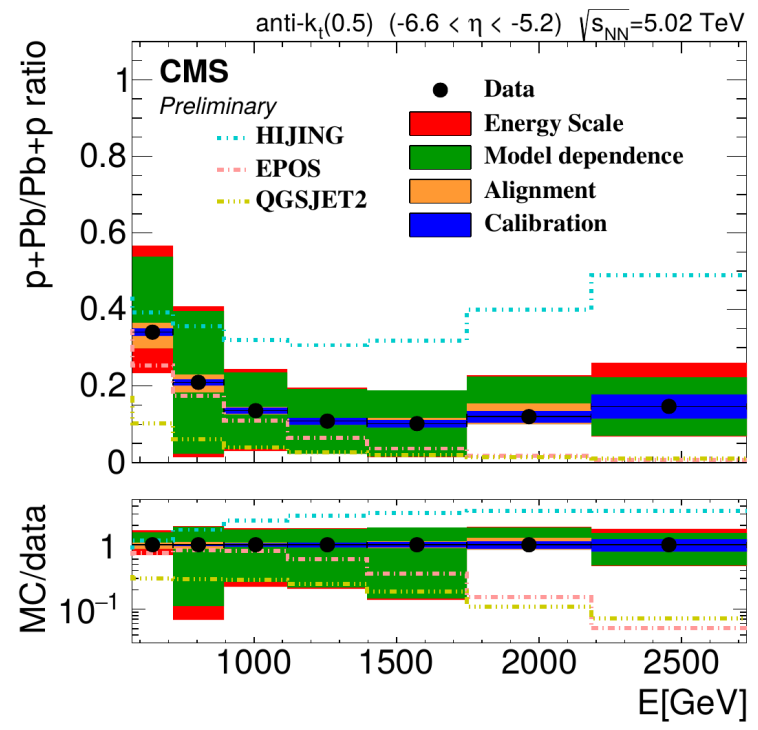

Figure 7. The ratio between proton-lead by lead-proton rates of very forward jets measured in CASTOR as function of the observed jet energy, see Ref. [10].
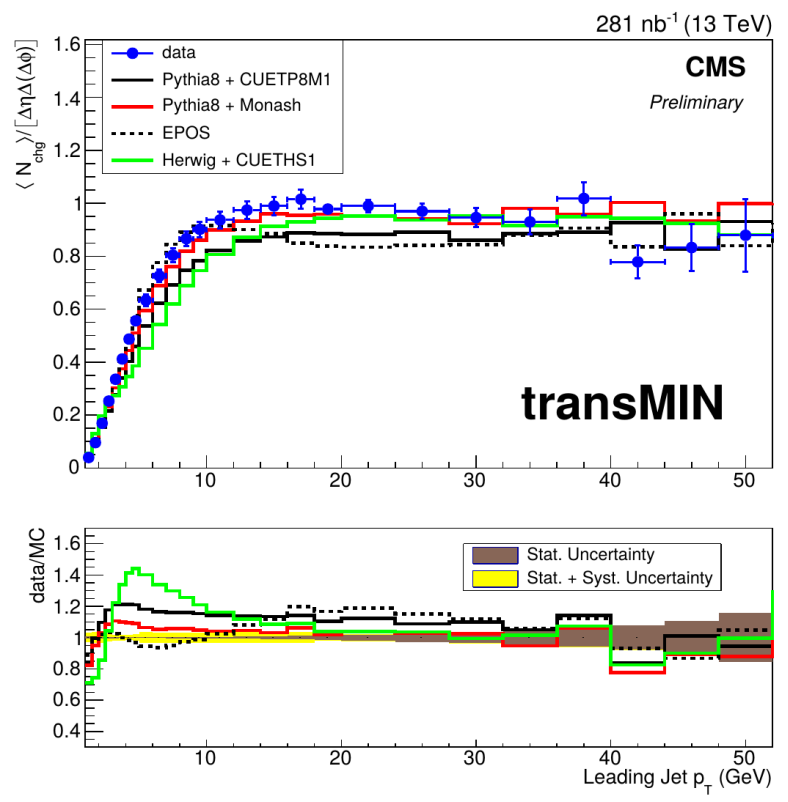

Figure 8. The average number of charged particles as function of the leading jet $p_{\mathrm{T}}$ in proton-proton collisions at $13 \mathrm{TeV}$, see Ref. [11]. Shown here is the "transMIN" region, which is transverse to the leading jet and in the hemisphere with less activity.

While typical underlying event measurements are performed relative to the highest jet $p_{\mathrm{T}}$, CMS has also measured it in reference the production of a $\mathrm{Z}$ boson [12]. This is shown in Fig. 9.

Another way to study the internal partonic structure in hadron collisions is to search for double parton scattering. In Fig. 10 the measurement of same-sign WW production in proton-proton collisions at $8 \mathrm{TeV}$ [13] is shown. The backgrounds in such a measurement are numerous, thus,

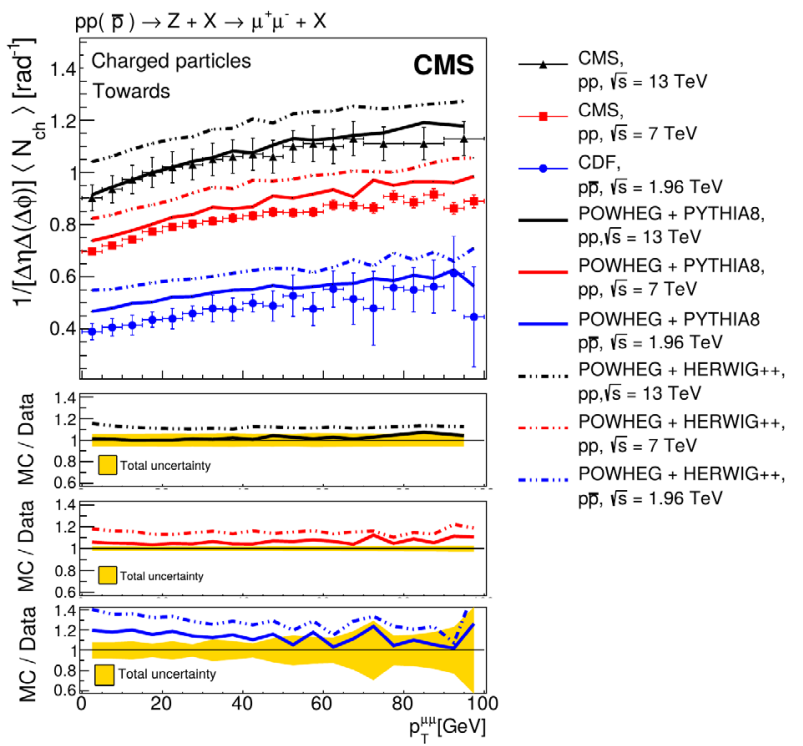

Figure 9. The average number of charged particles as function of central $\mathrm{Z}_{\mathrm{T}}$, see Ref. [12], in proton-proton collision of different centre-of-mass energies.

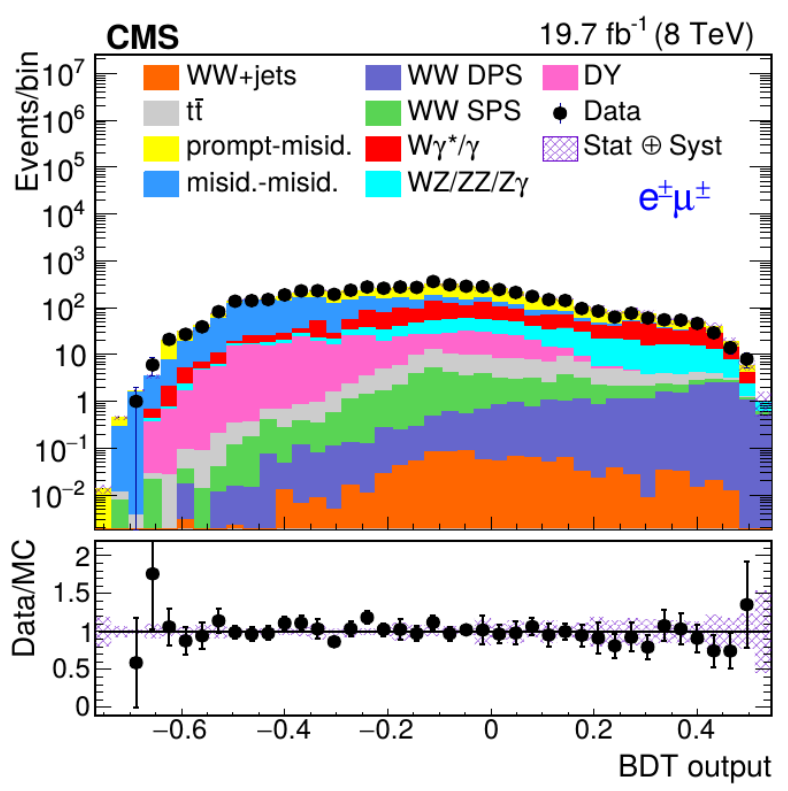

Figure 10. Output of a boosted decision tree for same-sign WW events in proton-proton collisions at $8 \mathrm{TeV}$. All relevant signal contributions are outlined. See Ref. [13].

only a multivariate analysis is able to extract the quantity of interest.

\section{Correlations}

It is an important discovery at the LHC that secondary particle production in proton-proton collisions contains a new type of correlations that were not expected. This was also observed in proton-proton collisions at $13 \mathrm{TeV}$ [15]. Such 

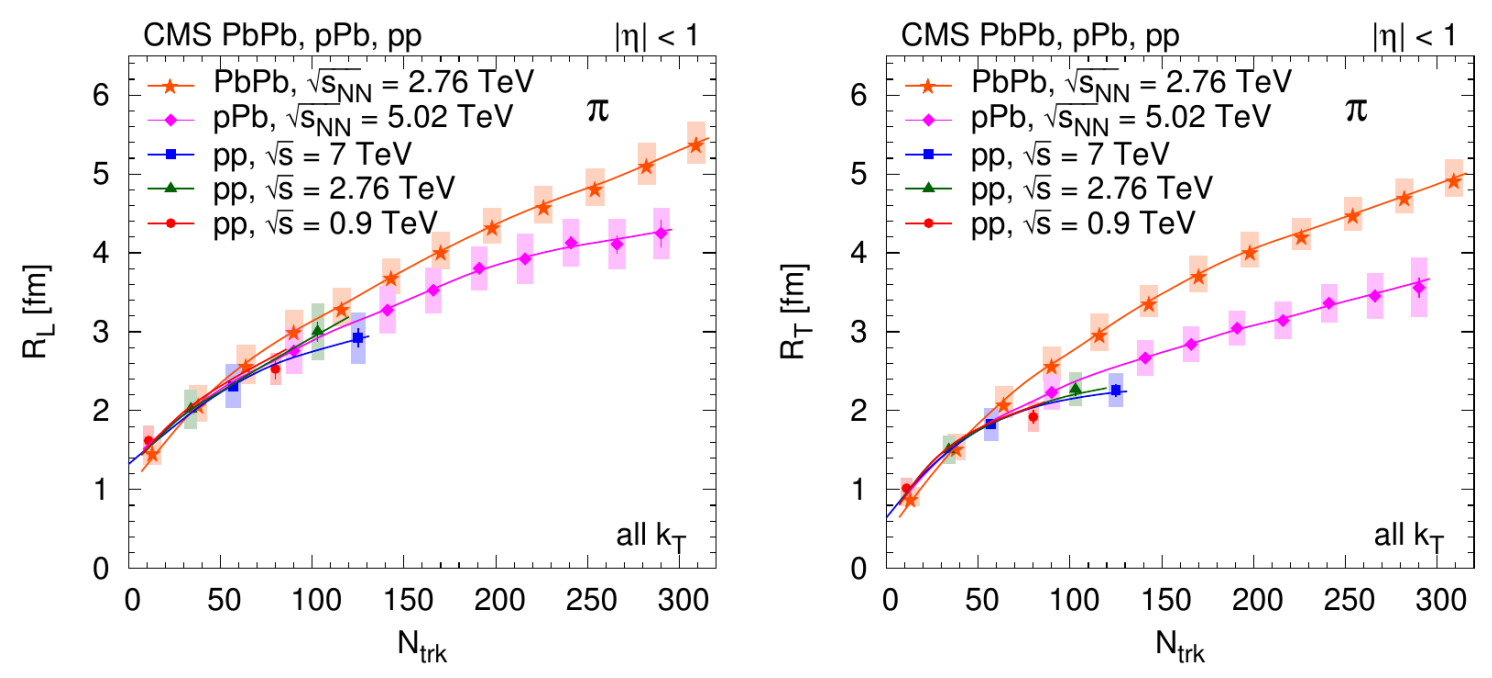

Figure 11. Geometry of the particle emission region measured in different collisions systems at different energies, see Ref. [14]. Shown are the parameters longitudinal size $R_{L}$ (left) and transverse size $R_{T}$ (right) as function of number of tracks, $N_{\text {trk }}$.

two-particle correlations were only known from heavyion collisions before. One possible conclusion from this could be that the underlying mechanisms in proton-proton, proton-lead and lead-lead collisions are the same and only depend on the maximum energy densities reached in collisions.

Another option to look at correlations is to observe Bose-Einstein correlations in the production of identical secondary particles. Due to interference effects, the probability to observe identical particles as a function of particle direction is modified. These data will contain information on the geometry of the emission region. In Fig. 11 some of these data are shown.

\section{Minimum bias results}

To describe very high energy cosmic ray interactions also minimum bias data is of particular importance. In cosmic ray interactions there are typically no filters or any sort of event selection. Thus the particle production in typical minimum bias collisions is what is most relevant. Here we will focus only on a single examples of this type of measurements.

One of the directly relevant results for very high cosmic ray interactions is the detailed measurements of the spectra of identified particles. In Fig. 12 some identified particle spectra determined in proton-proton collisions at $13 \mathrm{TeV}$ are shown. There is obvious room also by the EPOS-LHC model for improvements.

\section{Summary}

A brief overview of some of the most recent CMS forwardphysics measurements has been reported. The physics covered by those measurements covers a very wide and also diverse range of fields. Several unique and very important observations relevant for very high cosmic ray interactions are part of this.

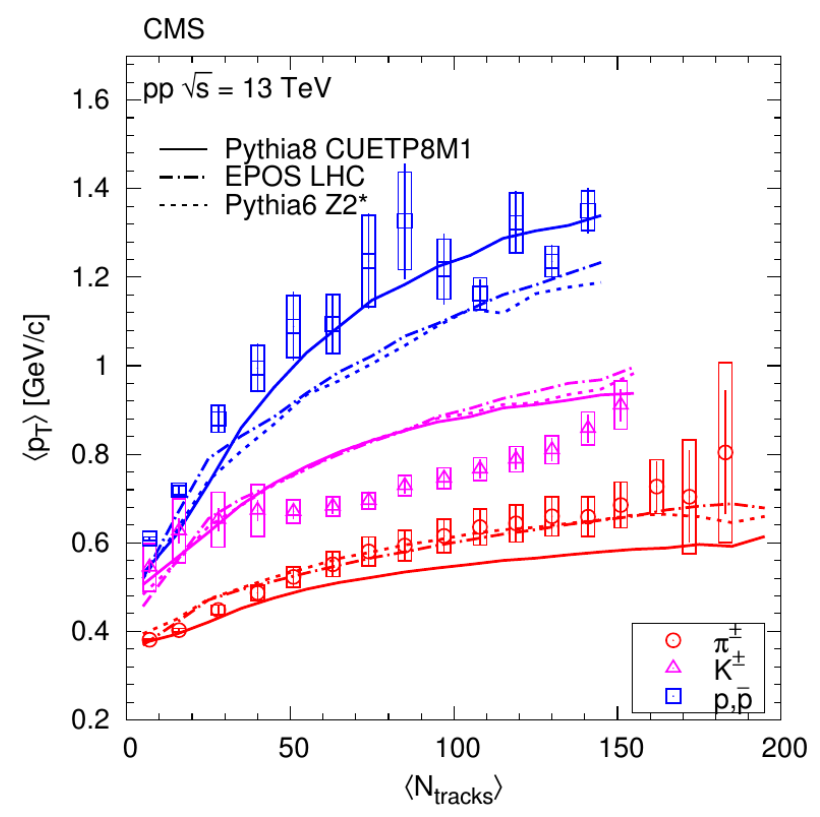

Figure 12. Measurement of the average $p_{\mathrm{T}}$ of identified particles versus the average number of charged tracks $N_{\text {tracks }}$ in protonproton collisions at $13 \mathrm{TeV}$, see Ref. [16].

\section{References}

[1] CMS Collaboration, JINST 3, S08004 (2008)

[2] CMS Collaboration, Eur. Phys. J. 79, 277 (2018), arxiv: 1809.11080 [hep-ex]

[3] CMS Collaboration, Submitted to: Phys. Rev. D (2017), arxiv: 1706.08310 [hep-ex]

[4] CMS Collaboration, Eur. Phys. J. C78, 242 (2018), arxiv: 1710.02586 [hep-ex]

[5] CMS Collaboration, JHEP 07, 161 (2018), arxiv: 1802.02613 [hep-ex] 
[6] CMS Collaboration, Phys. Rev. D92, 012003 (2015), arxiv: 1503.08689 [hep-ex]

[7] CMS Collaboration, JHEP 08, 119 (2016), arxiv: 1604.04464 [hep-ex]

[8] CMS and TOTEM Collaborations, JHEP 07, 153 (2018), arxiv: 1803.04496 [hep-ex]

[9] CMS Collaboration, JHEP 03, 156 (2017), arxiv: 1609.05331 [hep-ex]

[10] CMS Collaboration, Submitted to: JHEP (2018), arxiv: 1812.01691 [hep-ex]

[11] CMS Collaboration, CMS-PAS-15-007 (2015) http://cds. cern.ch/record/2104473 [hep-ex]

[12] CMS Collaboration, JHEP 07, 032 (2018), arxiv: 1711.04299 [hep-ex]

[13] CMS Collaboration, JHEP 02, 032 (2018), arxiv: 1712.02280 [hep-ex]

[14] A.M. Sirunyan et al. (CMS), Phys. Rev. C97, 064912 (2018), arxiv: 1712.07198 [hep-ex]

[15] V. Khachatryan et al. (CMS), Phys. Rev. Lett. 116, 172302 (2016), arxiv: 1510.03068 [hep-ex]

[16] A.M. Sirunyan et al. (CMS), Phys. Rev. D96, 112003 (2017), arxiv: 1706.10194 [hep-ex] 